\title{
A GENERALIZATION OF SELBERG'S BETA INTEGRAL
}

\author{
ROBERT A. GUSTAFSON
}

\begin{abstract}
We evaluate several infinite families of multidimensional integrals which are generalizations or analogs of Euler's classical beta integral. We first evaluate a $q$-analog of Selberg's beta integral. This integral is then used to prove the Macdonald-Morris conjectures for the affine root systems of types $S\left(C_{l}\right)$ and $S\left(C_{l}\right)^{\vee}$ and to give a new proof of these conjectures for $S\left(B C_{l}\right), S\left(B_{l}\right), S\left(B_{l}\right)^{\vee}$ and $S\left(D_{l}\right)$.
\end{abstract}

\section{INTRODUCTION}

In 1944, A. Selberg [23] evaluated the following integral (see also Aomoto [1]):

$$
\begin{aligned}
\int_{0}^{1} & \cdots \int_{0}^{1} \prod_{1 \leq i<j \leq n}\left|t_{i}-t_{j}\right|^{2 z} \prod_{j=1}^{n} t_{j}^{x-1}\left(1-t_{j}\right)^{y-1} d t_{j} \\
= & \prod_{j=1}^{n} \frac{\Gamma(x+(j-1) z) \Gamma(y+(j-1) z) \Gamma(j z+1)}{\Gamma(x+y+(n+j-2) z) \Gamma(z+1)},
\end{aligned}
$$

where $n$ is a positive integer, $x, y, z \in \mathbf{C}$ and $\operatorname{Re}(x), \operatorname{Re}(y)>0$ and $\operatorname{Re}(z)>-\max \left\{\frac{1}{n}, \operatorname{Re}(x) /(n-1), \operatorname{Re}(y) /(n-1)\right\}$. For $n=1$, the integral (1) reduces to Euler's classical beta integral.

Now let $n \geq 1$ and $a_{1}, a_{2}, a_{3}, a_{4}, b, q \in \mathbf{C}$ with

$$
\max \left\{\left|a_{1}\right|, \ldots,\left|a_{4}\right|,|b|,|q|\right\}<1 .
$$

For $c \in \mathbf{C}$ define

$$
[c ; q]_{\infty}=[c]_{\infty}=\prod_{k=0}^{\infty}\left(1-c q^{k}\right) .
$$

Received by the editors April 19, 1989.

1980 Mathematics Subject Classification (1985 Revision). Primary 33A15, 33A75, 05A 19.

This research is partially supported by NSF Grant INT-8713472. 
If $T^{n}$ is the $n$-fold direct product of the unit circle $\{t \in \mathbf{C}|| t \mid=1\}$ traversed in the positive direction, then we can evaluate the integral

$$
\begin{aligned}
& \frac{1}{(2 \pi i)^{n}} \int_{T^{n}} \prod_{1 \leq j<k \leq n} \frac{\left[t_{j} t_{k}^{-1}\right]_{\infty}\left[t_{j}^{-1} t_{k}\right]_{\infty}\left[t_{j} t_{k}\right]_{\infty}\left[t_{j}^{-1} t_{k}^{-1}\right]_{\infty}}{\left[b t_{\infty}^{-1}\right]_{\infty}\left[b t_{j}^{-1} t_{k}\right]_{\infty}\left[b t_{j} t_{k}\right]_{\infty}\left[b t_{j}^{-1} t_{k}^{-1}\right]_{\infty}} \\
& \cdot \prod_{j=1}^{n} \frac{\left[t_{j}^{2}\right]_{\infty}\left[t_{j}^{-2}\right]_{\infty} d t_{j}}{\prod_{k=1}^{4}\left\{\left[a_{k} t_{j}\right]_{\infty}\left[a_{k} t_{j}^{-1}\right]_{\infty}\right\} t_{j}} \\
& \quad=2^{n} n ! \prod_{j=1}^{n} \frac{[b]_{\infty}\left[b^{n+j-2} \prod_{k=1}^{4} a_{k}\right]_{\infty}}{\left[b^{j}\right]_{\infty}[q]_{\infty} \prod_{1 \leq k<l \leq 4}\left[a_{k} a_{l} b^{j-1}\right]_{\infty}}
\end{aligned}
$$

Then $n=1$ case of integral (2) is due to Askey and Wilson [4]. The integral (2) is a $q$-analog of (1) in the sense that after a change of variables and an appropriate specialization of (2) and limit as $q \rightarrow 1$, then (1) can be deduced from (2).

Selberg's integral (1) has had diverse applications in fields ranging from number theory, physics, statistics, combinatorics, algebra and analysis. Two particular applications were a use by Bombieri to prove Mehta's conjecture [18] and by Macdonald [17] to prove some of his conjectures $(q=1$ case) for the affine root systems (for definition and properties see [15]) of types $S\left(B C_{l}\right), S\left(B_{l}\right)$, $S\left(B_{l}\right)^{\vee}, S\left(C_{l}\right), S\left(C_{l}\right)^{\vee}$ and $S\left(D_{l}\right)$ for all $l \geq 1$ (when defined). Just as Macdonald used integral (1) to prove some of his $(q=1)$ conjectures, we will use integral (2) to prove for the same set of affine root systems the corresponding Macdonald-Morris conjectures with arbitrary parameter $q$.

Macdonald's root system conjectures in [17] were motivated partly by a conjecture of Dyson [7] related to the root system $A_{n}$, a $q$-analog of Dyson's conjecture made by Andrews [2] and some conjectures of Morris [19] for the root system of type $G_{2}$. Dyson's conjecture was proved by Gunson [10] and Wilson [25]. The Andrews-Dyson conjecture was proved by Zeilberger and Bressoud [28].

Morris' Conjecture A in [19] for arbitrary parameter $q$ and any reduced irreducible affine root system $S$ extends Macdonald's Conjectures 2.3 and 3.1 in [17]. In the simplest case of these Macdonald-Morris conjectures, let $R$ be a reduced finite (not affine) root system of rank $l$ with basis $\left\{\alpha_{1}, \ldots, \alpha_{l}\right\}$. For each $\alpha \in R$, let $e^{\alpha}$ be the formal exponential, which is an element of the group ring of the lattice generated by $R$. Let $d_{1}, \ldots, d_{l}$ 
be the degrees of the fundamental invariants of the Weyl group $W(R)$.

Conjecture (Macdonald [17, Conjecture 3.1]). With the above notation, the constant term (i.e. involving $q$ but no exponential $e^{\alpha}$ ) in

$$
\prod_{\alpha>0} \prod_{i=1}^{k}\left(1-q^{i-1} e^{-\alpha}\right)\left(1-q^{i} e^{\alpha}\right)
$$

where $k$ is a positive integer or $+\infty$ is

$$
\prod_{i=1}^{l}\left[\begin{array}{c}
k d_{i} \\
k
\end{array}\right]
$$

where

$$
\left[\begin{array}{l}
n \\
r
\end{array}\right]
$$

is the " $q$-binomial coefficient"

$$
\frac{\left(1-q^{n}\right)\left(1-q^{n-1}\right) \cdots\left(1-q^{n-r+1}\right)}{(1-q)\left(1-q^{2}\right) \cdots\left(1-q^{r}\right)} .
$$

We will actually prove the more general Morris' Conjecture A [19] for the affine root systems $S$ of types $S\left(B C_{l}\right), S\left(B_{l}\right)$, $S\left(B_{l}\right)^{\vee}, S\left(C_{l}\right), S\left(C_{l}\right)^{\vee}$ and $S\left(D_{l}\right)$ for all $l \geq 1$ (when defined) and for arbitrary parameter $q$. Macdonald's Conjecture 3.1 stated above, where $R$ is a finite root system of type $B_{l}, C_{l}$ or $D_{l}$, then follows as a special case of Morris' Conjecture A for $S\left(B_{l}\right), S\left(C_{l}\right)$ and $S\left(D_{l}\right)$. Kadell [14] has previously proved these conjectures for all affine root systems of type $S\left(B C_{l}\right)$ and hence $S\left(B_{l}\right), S\left(B_{l}\right)^{\vee}$ and $S\left(D_{l}\right)$. The Macdonald-Morris conjectures for $R=G_{2}$ have been proved by Habsieger [13] and Zeilberger [26]. See Garvan [8] for $F_{4}$, Garvan and Gonnet [9] for $S\left(F_{4}\right)^{\vee}$, Zeilberger [27] for $S\left(G_{2}\right)^{\vee}$ and Opdam [20] for the $q=1$ conjectures. There is also the conjecture of Rahman [21] which seems related to the special case of integral (2) where $a_{2}=q^{1 / 2} a_{1}$ and $a_{4}=q^{1 / 2} a_{3}$.

\section{Proof of integral (2)}

Since the $n=1$ case of (2) is proved in [4], we may assume that $n \geq 2$. Denote the integral on the left-hand side of (2) by $I_{n}\left(a_{1}, a_{2}, a_{3}, a_{4} ; b ; q\right)$. Let $c_{j} \in \mathbf{C},\left|c_{j}\right|<1$, for $1 \leq j \leq 2 n+2$ 
with $q$ and $T$ as above. In [11] we have evaluated the integral

$$
\begin{gathered}
\frac{1}{(2 \pi i)^{n}} \int_{T^{n}} \frac{\prod_{1 \leq j<k \leq n}\left\{\left[t_{j} t_{k}^{-1}\right]_{\infty}\left[t_{j}^{-1} t_{k}\right]_{\infty}\left[t_{j} t_{k}\right]_{\infty}\left[t_{j}^{-1} t_{k}^{-1}\right]_{\infty}\right\}}{\prod_{j=1}^{2 n+2} \prod_{k=1}^{n}\left[c_{j} t_{k}\right]_{\infty}\left[c_{j} t_{k}^{-1}\right]_{\infty}} \\
\cdot \prod_{j=1}^{n} \frac{\left[t_{j}^{2}\right]_{\infty}\left[t_{j}^{-2}\right]_{\infty} d t_{j}}{t_{j}} \\
=\frac{2^{n} n !\left[\prod_{j=1}^{2 n+2} c_{j}\right]_{\infty}}{[q]_{\infty}^{n}} \prod_{1 \leq j<k \leq 2 n+2}\left[c_{j} c_{k}\right]_{\infty}
\end{gathered}
$$

With notation as above, consider the integral

(4)

$$
\begin{aligned}
\frac{1}{(2 \pi i)^{2 n-1}} & \int_{T^{n}} \int_{T^{n-1}} \frac{\prod_{1 \leq j<k \leq n}\left\{\left[t_{j} t_{k}^{-1}\right]_{\infty}\left[t_{j}^{-1} t_{k}\right]_{\infty}\left[t_{j} t_{k}\right]_{\infty}\left[t_{j}^{-1} t_{k}^{-1}\right]_{\infty}\right\}}{\prod_{j=1}^{n} \prod_{k=1}^{4}\left[a_{k} t_{j}\right]_{\infty}\left[a_{k} t_{j}^{-1}\right]_{\infty}} \\
& \frac{\prod_{j=1}^{n}\left[t_{j}^{2}\right]_{\infty}\left[t_{j}^{-2}\right]_{\infty} \prod_{1 \leq j<k \leq n-1}\left\{\left[s_{j} s_{k}^{-1}\right]_{\infty}\left[s_{j}^{-1} s_{k}\right]_{\infty}\left[s_{j} s_{k}\right]_{\infty}\left[s_{j}^{-1} s_{k}^{-1}\right]_{\infty}\right\}}{\prod_{j=1}^{n} \prod_{k=1}^{n-1}\left\{\left[b^{1 / 2} s_{k} t_{j}\right]_{\infty}\left[b^{1 / 2} s_{k}^{-1} t_{j}\right]_{\infty}\left[b^{1 / 2} s_{k} t_{j}^{-1}\right]_{\infty}\left[b^{1 / 2} s_{k}^{-1} t_{j}^{-1}\right]_{\infty}\right\}} \\
& \cdot \prod_{k=1}^{n-1} \frac{\left[s_{k}^{2}\right]_{\infty}\left[s_{k}^{-2}\right]_{\infty} d s_{k}}{s_{k}} \prod_{j=1}^{n} \frac{d t_{j}}{t_{j}}
\end{aligned}
$$

where $b^{1 / 2}$ is any fixed square root of $b$. In the integral (4) we may use identity (3) to evaluate the interior integral either with respect to the set of variables $\left\{s_{1}, \ldots, s_{n-1}\right\}$ or, by changing the order of integration, with respect to the set of variables $\left\{t_{1}, \ldots, t_{n}\right\}$. Equating the resulting integrals we obtain

$$
\begin{aligned}
& \frac{2^{n-1}(n-1) !\left[b^{n}\right]_{\infty}}{[q]_{\infty}^{n-1}[b]_{\infty}^{n}} I_{n}\left(a_{1}, a_{2}, a_{3}, a_{4} ; b ; q\right) \\
& \frac{2^{n} n !\left[b^{n-1} \prod_{j=1}^{4} a_{j}\right]_{\infty}}{[q]_{\infty}^{n}[b]_{\infty}^{n-1} \prod_{1 \leq j<k \leq 4}\left[a_{j} a_{k}\right]_{\infty}} I_{n-1}\left(a_{1} b^{1 / 2}, \ldots, a_{4} b^{1 / 2} ; b ; q\right) .
\end{aligned}
$$


We finish the proof of identity (2) by doing induction on $n$, using identity (5) and the Askey-Wilson integral for the case $n=1$.

\section{Morris' Conjecture A}

We sketch a proof of Morris' Conjecture A [19] for the affine root systems $S$ of types $S\left(B C_{l}\right), S\left(B_{l}\right), S\left(B_{l}\right)^{\vee}, S\left(C_{l}\right), S\left(C_{l}\right)^{\vee}$ and $S\left(D_{l}\right)$ where $l \geq 1$ (when defined) and for arbitrary parameter $q$. The proof consists of specializing the parameters in identity (2) and making use of the identity found in Theorem 2.8 of [16]. As an illustration of this method of proof of Morris' Conjecture A, consider the case $S=S\left(C_{l}\right)$ where $l \geq 2$. Consider the integral $I_{l}\left(a^{1 / 2},-a^{1 / 2}, q^{1 / 2} a^{1 / 2},-q^{1 / 2} a^{1 / 2} ; b ; q\right)$ where $|a|,|b|<1$. Multiply the integrand in this integral by

$$
\prod_{1 \leq j<k \leq l} \frac{\left(1-b w\left(t_{j}^{-1} t_{k}\right)\right)\left(1-b w\left(t_{j}^{-1} t_{k}^{-1}\right)\right)}{\left(1-w\left(t_{j}^{-1} t_{k}\right)\right)\left(1-w\left(t_{j}^{-1} t_{k}^{-1}\right)\right)} \prod_{j=1}^{l} \frac{\left(1-a w\left(t_{j}^{-2}\right)\right)}{\left(1-w\left(t_{j}^{-2}\right)\right)},
$$

where $w$ is an element of the Weyl group $W$ of $C_{l}$, i.e. a permutation of the variables $t_{1}, \ldots t_{l}$ together with inversions $t_{j} \rightarrow t_{j}^{-1}$ and the corresponding action on $t_{1}^{-1}, \ldots, t_{l}^{-1}$. The resulting integral is independent of $w \in W$. Now summing over $w \in W$ and using the identity [16, Theorem 2.8] for $C_{l}$ we obtain

$$
\begin{aligned}
& \frac{1}{(2 \pi i)^{l}} \int_{T^{l}} \prod_{1 \leq j<k \leq l} \frac{\left[t_{j} t_{k}^{-1}\right]_{\infty}\left[q t_{j}^{-1} t_{k}\right]_{\infty}\left[t_{j} t_{k}\right]_{\infty}\left[q t_{j}^{-1} t_{k}^{-1}\right]_{\infty}}{\left[q t_{\infty}^{-1}\right]_{\infty}\left[q b t_{j}^{-1} t_{k}\right]_{\infty}\left[b t_{j} t_{k}\right]_{\infty}\left[q b t_{j}^{-1} t_{k}^{-1}\right]_{\infty}} \\
& \cdot \prod_{j=1}^{l} \frac{\left[t_{j}^{2}\right]_{\infty}\left[q t_{j}^{-2}\right]_{\infty} d t_{j}}{\left[a t_{j}^{2}\right]_{\infty}\left[q a t_{j}^{-2}\right]_{\infty} t_{j}} \\
& \quad=\prod_{j=1}^{l} \frac{[q b]_{\infty}\left[q a^{2} b^{l+j-2}\right]_{\infty}\left[q a b^{j-1}\right]_{\infty}^{2}}{[q]_{\infty}\left[q b^{j}\right]_{\infty}\left[q a^{2} b^{2(j-1)}\right]_{\infty}^{2}}
\end{aligned}
$$

which is equivalent to Morris' Conjecture A for $S\left(C_{l}\right)$ [19, p. 131]. Setting $a=b$ in (6), this also proves Macdonald's Conjecture 3.1 for $R=C_{l}$ as stated above.

\section{SOME INTEGRAL EVALUATIONS}

We state some integral identities whose proofs are similar to that of (2), making use of integral identities from [11 and 12]. 
Details of the proofs of these and realted integral identities should be given elsewhere.

Let $n \geq 1$ and $z_{1}, \ldots, z_{n}, \alpha_{1}, \ldots, \alpha_{4}, a_{1}, \ldots, a_{4}, \beta_{1}, \beta_{2}$, $b, \delta \in \mathbf{C}$ and $m_{1}, \ldots, m_{n} \in \mathbf{Z}$. Choose $z_{1}, \ldots, z_{n}$ so that the integrands in the integrals $(9)$ and (10) below have no poles. Then

(7)

$$
\begin{gathered}
\frac{1}{(2 \pi i)^{n}} \int_{-i \infty}^{i \infty} \cdots \int_{-i \infty}^{i \infty} \prod_{1 \leq j<k \leq n}\left\{\frac{\Gamma\left(\delta+t_{j}-t_{k}\right) \Gamma\left(\delta+t_{k}-t_{j}\right)}{\Gamma\left(t_{j}-t_{k}\right) \Gamma\left(t_{k}-t_{j}\right)}\right. \\
\left.\cdot \frac{\Gamma\left(\delta+t_{j}+t_{k}\right) \Gamma\left(\delta-t_{j}-t_{k}\right)}{\Gamma\left(t_{j}+t_{k}\right) \Gamma\left(-t_{j}-t_{k}\right)}\right\} \prod_{j=1}^{n} \frac{\prod_{k=1}^{4}\left\{\Gamma\left(\alpha_{k}+t_{j}\right) \Gamma\left(\alpha_{k}-t_{j}\right)\right\} d t_{j}}{\Gamma\left(2 t_{j}\right) \Gamma\left(-2 t_{j}\right)} \\
=2^{n} n ! \prod_{j=1}^{n} \frac{\Gamma(j \delta)}{\prod_{1 \leq k<l \leq 4} \Gamma\left(\alpha_{k}+\alpha_{l}+(j-1) \delta\right)} \\
\Gamma(\delta) \Gamma\left((n+j-2) \delta+\sum_{k=1}^{4} \alpha_{k}\right)
\end{gathered}
$$

where the contours of integration are the imaginary axis and

$$
\min \left\{\operatorname{Re}(\delta), \operatorname{Re}\left(\alpha_{1}\right), \ldots, \operatorname{Re}\left(\alpha_{4}\right)\right\}>0
$$

(8) $\frac{1}{(2 \pi i)^{n}} \int_{-i \infty}^{i \infty} \cdots \int_{-i \infty}^{i \infty} \prod_{\substack{1 \leq j, k \leq n \\ j \neq k}} \frac{\Gamma\left(\delta+t_{j}-t_{k}\right)}{\Gamma\left(t_{j}-t_{k}\right)}$

$$
\begin{aligned}
& \cdot \prod_{j=1}^{n}\left\{\prod_{k=1}^{2}\left[\left(\Gamma\left(\alpha_{k}+t_{j}\right) \Gamma\left(\beta_{k}-t_{j}\right)\right] d t_{j}\right\}\right. \\
& =n ! \prod_{j=1}^{n} \frac{\Gamma(j \delta) \prod_{k, l=1}^{2} \Gamma\left(\alpha_{k}+\beta_{l}+(j-1) \delta\right)}{\Gamma(\delta) \Gamma\left((n+j-2) \delta+\sum_{k=1}^{2}\left(\alpha_{k}+\beta_{k}\right)\right)},
\end{aligned}
$$

where the contours of integration are the imaginary axis and

$$
\min \left\{\operatorname{Re}(\delta), \operatorname{Re}\left(\alpha_{1}\right), \operatorname{Re}\left(\alpha_{2}\right), \operatorname{Re}\left(\beta_{1}\right), \operatorname{Re}\left(\beta_{2}\right)\right\}>0
$$


(9)

$$
\begin{aligned}
\int_{-\infty}^{\infty} & \cdots \int_{-\infty}^{\infty} \prod_{\substack{1 \leq j, k \leq n \\
j \neq k}} \frac{\Gamma\left(1+z_{j}+t_{j}-z_{k}-t_{k}\right)}{\Gamma\left(1+\delta+z_{j}+t_{j}-z_{k}-t_{k}\right)} \\
& \cdot \prod_{j=1}^{n} \frac{e^{2 \pi i m_{j} t_{j}} d t_{j}}{\prod_{k=1}^{2} \Gamma\left(1+\alpha_{k}+z_{j}+t_{j}\right) \Gamma\left(1+\beta_{k}-z_{j}-t_{j}\right)} \\
& =\left\{\begin{array}{c}
\prod_{j=1}^{n} \frac{\Gamma(1+\delta) \Gamma\left(1+(n+j-2) \delta+\sum_{k=1}^{2}\left(\alpha_{\kappa}+\beta_{\kappa}\right)\right)}{\Gamma(1+j \delta) \prod_{k, l=1}^{2} \Gamma\left(1+\alpha_{k}+\beta_{l}+(j-1) \delta\right)} \\
\text { if } m_{1}=\cdots=m_{n}=0
\end{array}\right.
\end{aligned}
$$

where

$$
\begin{aligned}
& \min \left\{\operatorname{Re}\left((n-1) \delta+\sum_{k=1}^{2}\left(\alpha_{k}+\beta_{k}\right)\right),\right. \\
& \left.\operatorname{Re}\left(2(n-1) \delta+\sum_{k=1}^{2}\left(\alpha_{k}+\beta_{k}\right)\right)\right\}>-1 \\
& \int_{-\infty}^{\infty} \cdots \int_{-\infty}^{\infty} \prod_{1 \leq j<k \leq n}\left\{\frac{\left[b q^{1+z_{j}+t_{j}-z_{k}}\right]_{\infty}\left[b q^{1-z_{j}-t_{j}+z_{k}+t_{k}}\right]_{\infty}}{\left[q^{1+z_{j}+t_{j}-z_{k}}\right]_{\infty}\left[q^{1-z_{j}-t_{j}+z_{k}+t_{k}}\right]_{\infty}}\right. \\
& \left.\cdot \frac{\left[b q^{1+z_{j}+t_{j}+z_{k}+t_{k}}\right]_{\infty}\left[b q^{1-z_{j}-t_{j}-z_{k}-t_{k}}\right]_{\infty}}{\left[q^{1+z_{j}+t_{j}+z_{j}+t_{k}}\right]_{\infty}\left[q^{1-z_{j}-t_{j}-z_{k}-t_{k}}\right]_{\infty}}\right\} \\
& \cdot \prod_{j=1}^{n} \frac{\left.\prod_{k=1}^{4}\left\{a_{k} q^{1+z_{j}+t_{j}}\right]_{\infty}\left[a_{k} q^{1-z_{j}-t_{j}}\right]_{\infty}\right\} \cdot e^{2 \pi i m_{j} t_{j}} d t_{j}}{\left[q^{1+2 z_{j}+2 t_{j}}\right]_{\infty}\left[q^{1-2 z_{j}-2 t_{j}}\right]_{\infty}} \\
& =\left\{\begin{array}{c}
\prod_{j=1}^{n} \frac{[q]_{\infty}\left[q b^{j}\right]_{\infty} \prod_{1 \leq k<l \leq 4}\left[q a_{k} a_{l} b^{j-1}\right]_{\infty}}{[q b]_{\infty}\left[q b^{n+j-2} \prod_{k=1}^{4} a_{k}\right]_{\infty}} \\
\text { if } m_{1}=\cdots=m_{n}=0
\end{array}\right.
\end{aligned}
$$


where

$$
\max \left\{\left|q b^{n-1} \prod_{k=1}^{4} a_{k}\right|,\left|q b^{2(n-1)} \prod_{k=1}^{4} a_{k}\right|\right\}<1
$$

and for simplicity we assume that $q \in \mathbf{R}, 0<q<1$. The $n=1$ case of (7) is due to de Branges [6] and Wilson [24], of (8) to Barnes [5], of (9) to Ramanujan [22] and (10) essentially to Askey [3].

Remarks. The integrals (9) and (10) are equivalent to multiple series summation theorems which generalize classical bilateral hypergeometric series summation theorems: Dougall's ${ }_{2} \mathrm{H}_{2}$ sum and Bailey's ${ }_{6} \psi_{6}$ sum. A similar connection between some related integral evaluations and the corresponding multiple series identities is explained in [12]. As we plan to describe elsewhere, we are led to conjecture a family of multiple series summation identities which are equivalent to the Macdonald-Morris conjectures and contain the Macdonald identities [15] as special cases.

\section{REFERENCES}

1. K. Aomoto, Jacobi polynomials associated with Selberg integrals, SIAM J. Math. Anal. 18 (1987), 545-549.

2. G. Andrews, Problems and prospects for basic hypergeometric functions, The Theory and Applications of Special Functions, (R. Askey, ed.), Academic Press, New York, 1975, pp. 191-224.

3. R. Askey, Beta integrals and q-extensions, Annamalai Univ. lecture, preprint.

4. R. Askey and J. Wilson, Some basic hypergeometric orthogonal polynomials that generalize Jacobi polynomials, Mem. Amer. Math. Soc., no. 319 (1985).

5. E. W. Barnes, A new development of the theory of hypergeometric functions, Proc. London, Math. Soc. (2) 6 (1908), 141-177.

6. L. de Branges, Tensor products spaces, J. Math. Anal. Appl. 38 (1972), 109148.

7. F. J. Dyson, Statistical theory of the energy levels of complex systems. I, J. Math. Phys. 3 (1962), 140-156.

8. F. Garvan, $A$ proof of the Macdonald-Morris root system conjecture for $F_{4}$, preprint.

9. F. Garvan and G. H. Gonnet, $A$ proof of the two parameter q-case of the Macdonald-Morris root system conjecture for $S\left(F_{4}\right)$ and $S\left(F_{4}\right)$ via Zeilberger's method, preprint.

10. J. Gunson, Proof of a conjecture of Dyson in the statistical theory of energy levels, J. Math. Phys. 3 (1962), 752-753.

11. R. Gustafson, Some q-beta and Mellin-Barnes integrals on compact Lie groups and Lie algebras, preprint.

12. __ Some multidimensional beta type integrals, preprint.

13. L. Habsieger La q-Macdonald-Morris pour $G_{2}$, C. R. Acad. Sci (Paris) 303 (1986), 211-213. 
14. K. Kadell, $A$ proof of the q-Macdonald-Morris conjecture for $B C_{n}$, preprint.

15. I. G. Macdonald, Affine root systems and Dedekind's $\eta$-function, Invent. Math. 15 (1972), 91-143.

16. _ , The Poincaré series of a Coxeter group, Math. Ann. 199 (1972), 161174.

17. _ Some conjectures for root systems, SIAM J. Math. Anal. 13 (1982), 988-1007.

18. M. L. Mehta, Random matrices and the statistical theory of energy levels, Academic Press, New York, 1967.

19. W. G. Morris, Constant term identities for finite and affine root systems: conjectures and theorems, $\mathrm{Ph}$. D. thesis, Univ. of Wisconsin-Madison, 1982.

20. E. Opdam, Some applications of hypergeometric shift operators, preprint.

21. M. Rahman, Another conjectured $q$-Selberg integral, SIAM J. Math. Anal. 17 (1986), 1267-1279.

22. S. Ramanujan, $A$ class of definite integrals, Quart. J. Math. 48 (1920), 294310.

23. A. Selberg, Bemerkinger om et multipelt integral, Norsk Mat. Tidsskr. 26 (1944), 71-78.

24. J. A. Wilson, Some hypergeometric orthogonal polynomials, SIAM J. Math. Anal. 11 (1980), 690-701.

25. K. Wilson, Proof of a conjecture of Dyson, J. Math. Phys. 3 (1962), 1040-1043.

26. D. Zeilberger, $A$ proof of the $G_{2}$ case of Macdonald's root system-Dyson conjecture, SIAM J. Math. Anal. 18 (1987), 880-883.

27. _ A Anified approach to Macdonald's root-system conjectures, SIAM J. Math. Anal 19 (1988), 987-1013.

28. D. Zeilberger and D. M. Bressoud, A proof of Andrews' q-Dyson conjecture, Discrete Math. 54 (1985), 201-224.

Department of Mathematics, Texas A\&M University, College Station, TEXAS 77843 
